\title{
Perspectives
}

\section{Sorbitol, Osmoregulation, and the Complications of Diabetes}

\author{
Maurice B. Burg and Peter F. Kador \\ National Heart, Lung and Blood Institute, and National Eye Institute, National Institutes of Health, Bethesda, Maryland 20892
}

\section{Introduction}

Sorbitol, glycerophosphorylcholine, inositol, and betaine are "compatible" or "nonperturbing" osmotically active organic solutes (organic "osmolytes"). These organic osmolytes accumulate to high levels in renal medullary cells in response to the elevated concentration of salt in the renal medullary interstitium (1).

Similar organic osmolytes accumulate in cells of a wide variety of organisms exposed to high salt environments (2). The organic osmolytes elevate intracellular osmolality to match the high external osmolality, while keeping the intracellular levels of sodium and potassium salts and cell volume near normal. High levels of sodium and potassium salts perturb intracellular macromolecules much more than do comparable levels of organic osmolytes. Thus, organic osmolytes help maintain the volume of cells and their internal milieu when they face a high and variable extracellular salt concentration. These compounds are also intermediates in a variety of biochemical pathways that are not obviously related to osmoregulation, and they occur in cells outside of the renal medulla. In view of their role in the renal medulla, it is plausible that they may also act as organic osmolytes in other tissues, but this has not been generally established.

In ocular and neural tissues increased levels of sorbitol along with concomitantly decreased levels of inositol are associated with the onset of diabetic complications $(3,4)$. Sorbitol is produced in cells from glucose by a reaction catalyzed by aldose reductase. In uncontrolled diabetes both the concentration of glucose in blood and the levels of aldose reductase increase in some tissues. Then, more sorbitol is produced. The increased sorbitol upsets osmoregulation in these cells and damages them. Inositol generally is actively transported into cells. Its lower level in diabetes appears to be linked to the higher levels of sorbitol and glucose. Low intracellular inositol has been associated with diabetic complications in nerve (4). Because of the experimental evidence that links increased levels of sorbitol with the onset of diabetic pathology, a series of clinical trials are underway with various aldose reductase inhibitors. The aim is to block the intracellular conversion of glucose to sorbitol and thus prevent some complications of diabetes.

The seemingly disparate studies on the renal medullary cells and on the complications of diabetes converge on the role of sorbitol in osmoregulation, both normal in the renal medul-

\footnotetext{
Address reprint requests to Dr. Burg, Building 10, Room 6N307, National Institutes of Health, Bethesda, MD 20892.

Received for publication 1 December 1987.
}

The Journal of Clinical Investigation, Inc.

Volume 81, March 1988, 635-640 lary cells, and abnormal in the neural and ocular tissues affected in diabetes.

\section{Organic osmolytes and osmoregulation}

Since cell membranes are incapable of sustaining significant osmotic pressure differences, the osmolality inside a cell must remain close to that of its surroundings. Therefore, when the extracellular salt concentration rises, intracellular solute concentration increases as well. This occurs in all animal cells, including those in the renal inner medulla. The initial response to increased salt in the environment is loss of water by osmosis, accompanied by an increase in the concentration of the solutes that remain behind in the cells. Many types of osmotically shrunken cells regulate back towards their normal volume by accumulating sodium and potassium salts, followed by uptake of water. However, this phase of osmoregulation is only temporary since in the long term, cells in general (2), and renal medullary cells in particular $(1,5)$, do not balance high extracellular salt concentrations with equally high intracellular concentrations of sodium and potassium salts. Instead, they accumulate organic osmolytes either by uptake from their surroundings or by synthesis. Thus, cellular osmoregulation, after an increase in extracellular salt concentration, occurs in two phases: $(i)$ volume regulatory increase by influx of sodium and potassium salts, followed by (ii) accumulation of organic osmolytes, replacing the sodium and potassium salts and thus restoring the intracellular milieu.

A theoretical basis for understanding organic osmolytes was first established in explaining the high level of amino acids in the cells of some euryhaline animals (2). This accumulation is restricted to neutral and acidic amino acids and is directly related to environmental salinity. The particular neutral and acidic amino acids that are accumulated resemble the cations and anions in the Hofmeister or lyotropic series that favor "native" macromolecular structure and function. In contrast, high concentrations of sodium and potassium chloride tend to denature macromolecules. Polyhydric alcohols, like sorbitol and inositol, are analogous in this respect to neutral and acidic amino acids (2). Osmotically active organic solutes with these properties are called "compatible solutes" or "nonperturbing solutes" (2).

The difference between perturbing and nonperturbing solutes is exemplified by their effects on enzyme function $(2,6)$. High levels of perturbing solutes, such as $\mathrm{NaCl}$ and $\mathrm{KCl}$, increase the $K_{\mathrm{m}}$ of some enzymes or decrease their $V_{\max }$, whereas even higher levels of nonperturbing solutes do not. In addition to enzymes, perturbing solutes also affect other types of proteins and nucleic acids (7) by altering the association-dissociation equilibria between macromolecules, the stability of macroscopic fibrous structures made up of proteins or nucleic acids, the conformation of nucleic acids and globular proteins, 
and the rates of macromolecular transconformational reactions.

Organic osmolytes, which are nonperturbing or compatible solutes, have been identified during water stress in a wide variety of bacteria, plants, and animals (2). They fall into three classes: ( $a$ ) polyhydric alcohols (polyols), $(b)$ free amino acids and their derivatives, and $(c)$ combinations of urea and methylamines. The predominant osmolytes in renal medullas fit these categories. Sorbitol and inositol are polyols, betaine is both an amino acid derivative and a methylamine, and glycerophosphorylcholine is both a polyol and methylamine $(1,5)$.

The concentration of organic osmolytes in renal medullary cells generally varies directly with the concentration of the urine $(1,8)$. The link presumably is the renal medullary extracellular salt concentration, which must be high to concentrate the urine and often varies with urine concentration. Thus, the accumulation of osmolytes in renal medullary cells must be regulated. Of the different organic osmolytes in the renal medulla, the control of sorbitol accumulation has been most completely studied.

\section{Regulation of sorbitol in renal medullary cells after changes in extracellular $\mathrm{NaCl}$ concentration}

In mammalian tissues sorbitol is produced from glucose in a reaction catalyzed by the enzyme aldose reductase (low $K_{\mathrm{m}}$ aldehyde reductase; alditol/NAD(P) ${ }^{+}$1-oxidoreductase, EC 1.1.1.21) (3): glucose + NADPH $\rightarrow$ sorbitol + NADP ${ }^{+}$. Sorbitol may in turn be oxidized by sorbitol dehydrogenase (1-iditol dehydrogenase, EC 1.1.1.14) to fructose (3). Sorbitol $+\mathrm{NAD}^{+} \rightarrow$ fructose $+\mathrm{NADH}$. This two-step conversion of glucose to fructose is known as the sorbitol or polyol pathway.

High levels of aldose reductase have been found in renal medullas (3). As determined by immunofluorescence microscopy with specific antibodies, aldose reductase is abundant in the terminal part of the collecting duct, thin limbs of Henle's loop, papillary surface epithelium, and interstitial cells. No staining was observed, however, in collecting ducts from the outer part of the inner medulla. Also, immunoreactive staining in the outer medulla was spotty and difficult to distinguish.

In rats deprived of water there is a gradient of sorbitol from $5 \mathrm{mmol} / \mathrm{kg}$ wet weight in the outer medulla to $\sim 60 \mathrm{mmol} / \mathrm{kg}$ wet weight at the tip of the papilla (8). The concentration of sorbitol in cell water is presumably even higher, because the sorbitol is mostly intracellular and the cell water is only a fraction of the wet weight. When rats (8) and rabbits (1) were given access to water the levels of sorbitol in the inner medulla decreased by $\sim 20-40 \%$. It is difficult to determine whether the change is consistent with the proposed role of sorbitol as an osmolyte since the resulting diuresis was modest, and the extent and duration of changes in medullary extracellular $\mathrm{NaCl}$ concentrations were not measured. It is difficult to accurately control and measure extracellular salt concentrations in the inner medulla in vivo. Therefore, the control of sorbitol levels and aldose reductase activity in renal cells has been most thoroughly investigated using cell culture.

GRB-PAP1 is a line of rabbit renal cells derived from the papillary surface epithelium (9), which is one of the renal medullary tissues that contains a high level of immunoreactive aldose reductase protein (3). These cells contain large amounts of aldose reductase and sorbitol when they are grown in hyperosmotic medium (500-600 mosmol/ $\mathrm{kg}$ ), but not when grown in medium with a normal osmolality (300 mosmol $/ \mathrm{kg})(10)$.
The intracellular sorbitol concentration was $240 \mathrm{mM}$ in the $600 \mathrm{mosmol} / \mathrm{kg}$ medium, which is sufficiently high to balance most of the $300 \mathrm{mosmol} / \mathrm{kg}$ excess of $\mathrm{NaCl}$ added to make the medium hyperosmotic. The cell water content and sodium and potassium concentrations in these cells did not differ significantly between the $300 \mathrm{mosmol} / \mathrm{kg}$ medium and the 600 mosmol/kg medium. Thus, the accumulation of sorbitol apparently increased the intracellular solute concentration enough to balance the high external osmolality, while the cells maintained normal volume and concentrations of sodium and potassium salts. No significant amounts of osmolytes other than sorbitol were identified. The increase in aldose reductase activity in hyperosmotic medium is due to an increase in the amount of enzyme protein (11). The enzyme, which has a weight of $39 \mathrm{kD}$ in this tissue, increased from low levels under isosmotic conditions to $>10 \%$ of the soluble cell protein in $600 \mathrm{mosmol} / \mathrm{kg}$ medium.

Large amounts of sorbitol produced by the cells appear in the medium (12). The amount of sorbitol in the medium after $24 \mathrm{~h}$ approximately equals the amount present in the cells at any given time. Because of the relatively large volume of medium, however, the concentration of sorbitol in the medium remains $<1 \mathrm{mM}$, which is much lower than the concentration in the cells. Initially, the large amounts of sorbitol in the medium seemed surprising because sorbitol is generally regarded as a nonpenetrating solute. Moreover, high cellular permeability to sorbitol would make sorbitol ineffective as an osmolyte. Considering the large concentration gradient and the long time involved ( $24 \mathrm{~h})$, it can be readily calculated, however, that the permeability to sorbitol actually is quite low.

The time course for changes in aldose reductase levels in GRB-PAP1 cells is relatively slow. When the medium was switched from 300 to 500 mosmol/kg by adding $\mathrm{NaCl}$, a time lag of 12-24 h occurred before aldose reductase activity and sorbitol concentrations began to increase. The increase was half maximal after $2 \mathrm{~d}$, and the highest levels were reached only after 3 to $4 \mathrm{~d}$ (13). When GRB-PAP1 cells were grown in a hyperosmotic medium ( $600 \mathrm{mosmol} / \mathrm{kg}$ ), and then switched to normal osmolality, the rate of decrease in aldose reductase was even slower. Both the amounts of aldose reductase protein and the enzyme activity decreased by only one-half in a week and required 2 wk to reach low levels (12).

In contrast to the slow fall in aldose reductase, sorbitol concentration fell rapidly in cells switched from 600 mosmol/ $\mathrm{kg}$ to $300 \mathrm{mosmol} / \mathrm{kg}$ medium. After medium osmolality was reduced, cell sorbitol decreased within $1 \mathrm{~d}$ to $10 \%$ of its initial value. This fall in cell sorbitol is accounted for by the accelerated efflux of sorbitol from the cells to the medium. This initial flux is very rapid and a large fraction of the cell sorbitol appears in the medium within 5 min after the osmolality is decreased (Bagnasco, S., and M. Burg, unpublished observation). Thus, changes in cell sorbitol in response to changes in medium osmolality occur by at least two mechanisms: slow change in aldose reductase activity and a rapid change in sorbitol flux.

The nature of the stimulus that induces aldose reductase in GRB-PAP1 cells was investigated by increasing the osmolality of the medium to the same extent with different solutes and comparing the magnitude of the changes. The effectiveness of the solutes depended on their molecular size (13). Raffinose, a trisaccharide with a large molecular size, had essentially the same effect as $\mathrm{NaCl}$. On the other hand urea and glycerol, both 
molecules with small molecular weights, did not cause increases in aldose reductase or sorbitol. The result apparently depends on the osmotic effectiveness of the solutes. Hyperosmotic addition of $\mathrm{NaCl}$ or raffinose causes prolonged shrinkage of the cells, associated with increased intracellular $\mathrm{Na}$ and $\mathrm{K}$ concentration. Urea, on the other hand, does not change either cell volume or the concentration of $\mathrm{Na}$ or $\mathrm{K}$ (except very transiently) because it exerts little osmotic effect due to its rapid penetration. Ouabain, which generally increases cell sodium, decreases cell potassium, and causes cells to swell, was used to perturb the relations between cell potassium, sodium, and water after increase in osmolality. Under those conditions, aldose reductase activity and sorbitol accumulation were not significantly related to cell sodium or water content. They were somewhat correlated with cell potassium, but most strongly related to the sum of cell sodium plus potassium concentration, i.e., ionic strength (Uchida, S., and M. Burg, unpublished observation). In this respect it is interesting to note that elevated cell potassium and ionic strength have been linked with high medium osmolality and increased transcription of the osmoregulatory proU gene, which codes for a betaine transporter, in Escherichia coli (14).

\section{Role of sorbitol and other sugar alcohols (polyols) in the complications of diabetes}

Although the accumulation of sorbitol may be beneficial for renal medullary cells, sorbitol and other polyols do not normally accumulate to high levels in other tissues, and when they do, cellular pathology may result. The best evidence for this is the fact that damage occurs in tissues that accumulate the polyol galactitol when animals are fed galactose, and this damage is preventable by aldose reductase inhibitors. Further, there is increasing experimental evidence that aldose reductase-dependent accumulation of sorbitol is a common biochemical link in the pathogenesis of many late-onset diabetic complications that are related to the control of blood glucose level $(3,15,16)$. The specific mechanisms by which the accumulation of polyols lead to the onset of these diabetic complications are the subject of continued investigation.

\section{Polyols and sugar cataracts}

The adverse effects of sorbitol were first observed by Kinoshita in the lens (17). In this tissue, when extracellular glucose concentration rises, more sorbitol is produced, and is catalyzed by an increased level of aldose reductase. The accumulation of sorbitol leads to cell swelling, which in turn leads to altered lens membrane permeability and biochemical changes associated with cataract formation. These observations led to the development of the osmotic hypothesis, which states that lens cell swelling in response to the aldose reductase-initiated accumulation of polyols leads to loss of cellular integrity.

The train of events is as follows (18). Accumulation of sorbitol raises intracellular osmolality, which results in an influx of water into the lens. Cell swelling is accompanied by increased membrane permeability, increased intracellular sodium, and decreased cell potassium. In addition, the levels of reduced glutathione, myo-inositol, ATP, and free amino acids decrease, and vacuoles form. As vacuole formation progresses, cortical opacification results. Protein synthesis decreases and the cells lose dry weight. Eventually, complete loss of osmotic integrity occurs. Electrolytes, amino acids, and proteins freely permeate the cell plasma membranes, and lens opacification proceeds to the final nuclear cataract stage.

The osmotic hypothesis is supported by a variety of experimental observations including in vitro lens culture studies, studies with various animal models, and the use of aldose reductase inhibitors that prevent or significantly delay cataract formation in proportion to their potency in preventing polyol formation in the lens (18). A variety of structurally diverse aldose reductase inhibitors have been used $(15,16,18)$. Of these, perhaps the best-known inhibitors undergoing clinical trials are the hydantoin sorbinil and the carboxylic acids Aldrestat (tolrestat), Ponalrestat (Statil), and Epalrestat (ONO 2235). Administered either orally, by injection, or topically as eye drops, these inhibitors delay or prevent the appearance of diabetic or galactosemic (sugar) cataracts in animals. Animal studies demonstrate that the rate of sugar cataract formation is directly dependent upon both the levels of aldose reductase present in the lens and on how well the sugar serves as a substrate for this enzyme (18). Cataracts occur in galactose-fed animals because aldose reductase reduces a variety of aldonic sugars, including galactose. High levels of galactose cause faster and greater damage than do high levels of glucose because galactose is a better substrate for aldose reductase, and thus is reduced to galactitol (dulcitol) more rapidly than glucose is to sorbitol. Further, galactitol is more persistent than sorbitol because galactitol is not further metabolized by sorbitol dehydrogenase. In addition to cataract formation, galactose-fed animals also display other cellular pathology similar to diabetes, except that the pathology occurs earlier under galactosemic conditions and is more severe than in the diabetic state. This has been useful in elucidating the relationship between aldose reductase and diabetic complications because the galactosemic animals have normal insulin levels.

Aldose reductase activity and immunohistochemical staining of aldose reductase protein increase in diabetic rat lenses, suggesting that the aldose reductase protein level is increased (19), just as it is in renal medullary cells exposed to high $\mathrm{NaCl}$. Aldose reductase activity is also increased in diabetic human lenses cultured in vitro, and these lenses accumulate higher levels of polyols than those from nondiabetics (18). Moreover, increased immunohistochemical staining for aldose reductase has also been observed in both the anterior and posterior superficial cortical layers of cataractous lenses extracted from diabetics, but not in those extracted from nondiabetics (19).

\section{Polyol effects in the cornea}

Aldose reductase is present in both the corneal epithelium and endothelium, and experimental studies link deranged osmoregulation in both regions to damage that occurs in diabetic and galactose-fed animals. Defects in the rate of reepithelialization occur in severely diabetic or galactosemic rats whose corneas have been denuded by limbus to limbus scraping ( 3 , $16,20)$, and the resurfaced corneas of these rats appear hazy and edematous. In addition, the epithelial cells appear distended and lack filopodia. Both the delay in reepithelialization and the hazy, edematous appearance of the cornea are prevented by oral or topical administration of aldose reductase inhibitors.

In the corneal endothelium, which consists of a monolayer of hexagonally shaped cells, there are changes in endothelial cell size (polymegathism) and shape (pleomorphism) in diabetic humans, rats, and dogs $(8,21,22)$. The diabetic endothe- 
lial monolayer may loose cells, and the remaining cells may have to stretch and slide to cover the cell-free areas. Topical administration of an aldose reductase inhibitor to diabetic rats has been reported to significantly reduce the extent of these morphological changes.

The clinical manifestations of these defects are decreased corneal sensitivity and decreased tolerance of the corneal epithelium to stress during therapies that include photocoagulation, vitrectomy, and even use of contact lenses. Traumatized areas of the corneal epithelium tend to heal more slowly in diabetics and require increased medical attention. Corneal thickening and persistent stromal edema, suggestive of abnormal endothelial cell function, have also been observed clinically. Limited administration of aldose reductase inhibitors in the United States and Japan has resulted in apparent improvement in the state of the corneal epithelium in diabetics $(23,24)$.

\section{Polyols and diabetic retinopathy}

Retinopathy leads to loss of vision in a significant fraction of diabetics. It is characterized by changes in the retinal capillary bed with initial formation of microaneurysms, exudates, and small intraretinal hemorrhages. Later, neovascularization, fibrovascular proliferation, and vitreous hemorrhages occur. Although the etiology of this complication remains uncertain, considerable evidence implicates the abnormal accumulation of polyols, which causes degeneration of retinal capillary pericytes. Trials are underway with aldose reductase inhibitors in an attempt to prevent retinopathy.

A distinguishing feature of early retinal changes is selective degeneration of pericytes (mural cells), whose long processes encircle the retinal endothelial capillary cells. Loss of pericytes is associated with decreased capillary tonicity, followed by formation of microaneurysms, dilation of vessels, increased number of endothelial cells, and formation of some acellular vessels. There is evidence that sorbitol accumulates in pericytes and causes them to degenerate into cell ghosts. Aldose reductase protein (detected immunohistochemically) is present in the mural cells but not in the endothelial cells of human and dog retinal capillaries $(16,25)$. Cultured human and dog retinal mural cells display aldose reductase activity, and the production of polyol in these cells is inhibited by aldose reductase inhibitors (26). Finally, retinal vascular changes similar to those observed in diabetic dogs and humans occur in galactose-fed dogs (27). Galactitol production in isolated dog retinal vessels cultured in medium containing $30 \mathrm{mM}$ galactose is significantly reduced by the aldose reductase inhibitor sorbinil (28).

In addition to pericyte degeneration, retinal capillary basement membrane thickening is commonly observed in diabetic animals and those fed galactose. This thickening can be prevented by aldose reductase inhibitors (29). The relationship between aldose reductase and basement membrane thickening remains unclear. Possibly, the membrane thickening is secondary to degeneration of the retinal pericytes.

Because of the considerable evidence linking retinopathy to polyol accumulation, a number of clinical studies are being conducted to see if aldose reductase inhibitors can delay or arrest the progression of retinal changes in early diabetic retinopathy. No conclusive results are reported, as yet. However, a possible indication of clinical improvement is the report that sorbinil reduces the blood retinal barrier permeability in diabetics, as monitored by vitreous fluorophotography (30).

\section{Diabetic neuropathy}

A variety of neurological disorders are associated with diabetes mellitus, and a majority of all diabetics are to some degree afflicted with neurological symptoms. Recent reviews have discussed the relationship of aldose reductase to neurological symptoms $(3,16)$.

Both sorbitol and myo-inositol appear to be involved in the onset of neuropathy, and their relative roles are questioned. The possible primacy of myo-inositol has been emphasized elsewhere (4); the relationship to sorbitol accumulation will mainly be considered in the present context. In peripheral nerves such as the sciatic, there is aldose reductase in Schwann cells, which are the cells responsible for the formation and maintenance of the myelin sheath $(3,16)$. Sorbitol and galactitol accumulate in the sciatic nerves of diabetic and galactosemic rats, respectively, and cause these nerves to swell. This occurs more rapidly in the galactose-fed than in the diabetic rats, and can be reversed by aldose reductase inhibitors (31, 32). These changes resemble those that have been shown to damage lenses (16). Polyol accumulation and nerve swelling are associated with decreased nerve myo-inositol and amino acid levels, decreased nerve conduction velocity, and decreased choline acetyltransferase. Polyol accumulation and the other effects are prevented by aldose reductase inhibitors (3, $16,33,34)$.

Impairment of motor nerve conduction, a clinical hallmark of peripheral neuropathy, is among the earliest and most easily quantifiable signs of diabetic neuropathy. Clinical improvement in nerve conduction has been reported with several aldose reductase inhibitors. A small but significant increase in motor nerve conduction was first reported in a multicenter randomized, doubly masked, crossover trial with sorbinil (35). This has been followed by other studies, many with inconclusive results (16). Nevertheless, the positive effects of sorbinil treatment, as reported in the initial multicentered trial, were also supported by morphological and morphometric examinations of sural nerve biopsies (36). Several studies indicate more positive responses in patients with mild neuropathy compared with those with severe neuropathy. Perhaps, aldose reductase inhibitors improve nerve conduction velocities in patients with mild, but not severe, neuropathy.

Aldose reductase inhibitors may be useful for the subjective relief of pain. First observed in early clinical trials with alrestatin (37), this has become more evident in recent studies with sorbinil, which is more potent. In the sorbinil trials patients who were unresponsive to conventional therapy experienced decreased pain, improved sensory perception, increased muscle strength, and normalization of nerve conduction velocities $(38,39)$.

The initial clinical trials with alrestatin (37) also suggested that aldose reductase inhibitors may be beneficial for symptoms of autonomic neuropathy, which include gastroparesis, diarrhea, erectile dysfunction, abnormal sudomotor, pupillary function, and weakness. Since then, these symptoms have all been reported to qualitatively improve in selected patients receiving sorbinil $(16,39)$. Sorbinil also produced quantitative improvements in the ratio of the R-R interval during expiration to that during inspiration (E/I ratio) and decreases in the 
minimal heart rate $(16,39)$. Beneficial effects of sorbinil on cardiac performance have also been reported (40).

\section{Do aldose reductase and sorbitol have a normal osmoregulatory role in nonrenal tissues affected by the complications of diabetes?}

In view of the evidence that aldose reductase and sorbitol help regulate the volume and intracellular milieu of renal medullary cells after changes in external osmolality, it is worth considering whether they have a similar role in the lens and other tissues. Recently, based on indirect evidence, it was proposed that aldose reductase activity and intracellular sorbitol accumulation protect lenses against daily, diet- and disease-related changes in osmotic pressure in the aqueous humor (41). This theory was tested by incubating rabbit lenses for $4 \mathrm{~h}$ in medium made hyperosmotic with added glucose $(55.5 \mathrm{mM})(42)$. Sorbitol and fructose accumulated continuously during the hyperosmotic period, but never reached a level high enough to balance the hyperosmolality of the glucose added to the medium. Then, the glucose and osmolality were reduced to normal levels for four additional hours. Lens sorbitol and fructose remained elevated at approximately a constant level during this time. The authors concluded that the observed accumulation of sorbitol and fructose was beneficial for reducing dehydration of the lens during acute cyclical changes in blood glucose, but that continuous, rather than cyclical activity of the pathway might be detrimental and cause cataracts. The putative acute osmoregulatory benefit of the sorbitol and fructose may be questioned, however, considering that they accumulated in the lenses slowly (42), and that their level did not fall when the glucose was reduced to isosmotic levels. In this respect renal medullary cells were strikingly different. Recall that a large efflux of sorbitol reduced its level in PAP-HT25 cells within minutes after the medium osmolality was decreased (9). Based on these observations, the role of aldose reductase and sorbitol in osmoregulation may well differ between renal and lens cells, but an exact comparison is difficult because the experimental conditions were so different in the various studies. In addition, it is not clear whether hyperosmolality associated with hyperglycemia causes the increase in aldose reductase activity observed in lenses of diabetic rats analogous to the effect of hyperosmolality in renal medullary cells.

\section{Summary and conclusions}

Sorbitol, whose synthesis is catalyzed by aldose reductase, is one of several organic solutes that renal medullary cells use to osmoregulate in the face of high and variable osmolality in the renal medullary extracellular fluid. Evidently, sorbitol accumulation is beneficial to renal medullary cells, and they are able to control its level without obvious harm. There is no such obvious normal role for the aldose reductase and sorbitol which occur in lenses, corneas, retinal vessels, and peripheral nerves, and there is considerable evidence that accumulation of sorbitol in these tissues during uncontrolled diabetes causes abnormal osmoregulation and subsequent cellular pathology. Aldose reductase inhibitors may prevent harmful sorbitol accumulation in diabetes. The possible helpfulness of these drugs in preventing complications of diabetes is being tested in clinical trials. Some benefit has been demonstrated in a limited number of patients with diabetic neuropathy and corneal erosions. However, clinical trials studying these and other com- plications, such as retinopathy and cataracts, are unfinished, and will require years to complete because of the chronic and erratic progression of these complications. Nonetheless, there is considerable promise based on these early trials and on well-documented studies in animals, and there is reason to hope that aldose reductase inhibitors will prove clinically useful for preventing at least some of the distressing complications of diabetes.

\section{References}

1. Bagnasco, S., R. Balaban, H. M. Fales, Y.-M. Yang, and M. Burg. 1986. Predominant osmotically active organic solutes in rat and rabbit renal medullas. J. Biol. Chem. 261:5872-5877.

2. Yancey, P. H., M. E. Clark, S. C. Hand, R. D. Bowlus, and G. N. Somero. 1982. Living with water stress: evolution of osmolyte systems. Science (Wash. DC). 217:1214-1222.

3. Dvornik, D. 1987. Aldose Reductase Inhibition: An Approach to the Prevention of Diabetic Complications. McGraw-Hill, Inc., New York.

4. Green, D. A., S. A. Lattimer, and A. A. F. Sima. 1987. Sorbitol, phosphoinositidesk and sodium-potassium-ATPase in the pathogenesis of diabetic complications. N. Engl. J. Med. 316:599-605.

5. Balaban, R. S., and M. B. Burg. 1987. Osmotically active organic solutes in the renal inner medulla. Kidney Int. 31:562-564.

6. Somero G. N. 1986. Protons, osmolytes, and fitness of internal milieu for protein function. Am. J. Physiol. 251:R197-R213.

7. Von Hippel, P. H., and T. Schleich. 1969. The effects of neutral salts on the structure and conformational stability of macromolecules in solution. In Structure and Stability of Biological Macromolecules. S. N. Timasheff and G. D. Fasman, editors. Marcel Dekker, Inc., New York. 417-574.

8. Oates, P. J., and K. J. Goddu. 1987. A sorbitol gradient in rat inner medulla. Kidney Int. 31:448.

9. Uchida, S., N. Green, H. Coon, T. Triche, S. Mims, and M. Burg. 1987. High $\mathrm{NaCl}$ induces stable changes in phenotype and karyotype of renal cells in culture. Am. J. Physiol. 253(Cell Physiol. 22):C230-C242.

10. Bagnasco, S. M., S. Uchida, R. S. Balaban, P. F. Kador, and M. B. Burg. 1987. Induction of aldose reductase and sorbitol in renal inner medullary cells by elevated extracellular $\mathrm{NaCl}$. Proc. Natl. Acad. Sci. USA. 84:1718-1720.

11. Bedford, J. J., S. M. Bagnasco, P. Kador, H. W. Harris, and M. B. Burg. 1987. Characterization and purification of a mammalian osmoregulatory protein, aldose reductase, induced in renal medullary cells by high extracellular NaCl. J. Biol. Chem. 262:14255-14259.

12. Bagnasco, S., J. Bedford, and M. Burg. 1987. Slow changes in aldose reductase and rapid changes in sorbitol flux mediate osmoregulation by renal medullary cells. Fed. Proc. 46:1229. (Abstr.)

13. Uchida, S., A. Garcia-Perez, and M. Burg. 1987. Mechanism by which high $\mathrm{NaCl}$ induces aldose reductase activity in cells cultures from kidney medulla. Fed. Proc. 46:1229. (Abstr.)

14. Higgins, F. H., J. Cairney, D. A. Stirling, L. Sutherland, and I. R. Booth. 1987. Osmotic regulation of gene expression: ionic strength as an intracellular signal? Trends Biolog. Science. 12:339-344.

15. Kador, P. F., N. E. Sharpless, and J. H. Kinoshita. 1985. Perspective series: aldose reductase inhibitors: a potential new class of agents for the pharmacological control of certain diabetic complications. J. Med. Chem. 28:841-849.

16. Kador, P. F. 1987. The role of aldose reductase in the development of diabetic complications. Med. Res. Rev. In press.

17. Kinoshita, J. H. 1974. Mechanism initiating cataract formation. Invest. Ophthalmol. 13:713-724.

18. Kador, P. F., and J. H. Kinoshita. 1984. Diabetic galactosemic cataracts. Ciba Foundation Symposium No. 106. Human Cataract Formation. 106:110. 
19. Akagi, Y., P. F. Kador, and J. H. Kinoshita. 1986. Immunohistochemical localization for aldose reductase in diabetic lenses. Invest. Ophthalmol. Visual Sci. 28:163-167.

20. Datiles, M. B., P. F. Kador, H. N. Fukui, T. S. Hu, and J. H. Kinoshita. 1983. Corneal re-epithelialization in galactosemic rats. Invest. Ophthalmol. Visual Sci. 24:563-569.

21. Schultz, R. O., M. Matsuda, R. W. Yee, H. F. Edelhauser, and K. J. Schultz. 1984. Corneal endothelial changes in type I and type II diabetes. Am. J. Ophthalmol. 98:401.

22. Matsuda, M., T. Awata, Y. Ohashi, M. Inaba, M. Fukuda, and M. Reizo. 1987. The effects of aldose reductase inhibition on endothelial cell morphology in diabetic rats. Current Eye Res. 6:391-392.

23. Cobo, L. M. 1984. Aldose reductase and diabetic keratopathy. In Aldose Reductase and Complications of Diabetes. Ann. Intern. Med. 101:87.

24. Ohashi, Y., T. Mano, H., Umemoto, R. Hosotani, Y. Tamada, Y. Tano, M. Matsuda, M. Fukuda, and R. Manabe. Effects of aldose reductase inhibitor (CT-112) on diabetic keratoepitheliopathy. Exerpta Med. Congr. Ser. In press.

25. Akagi, Y., H. Terubayashi, J. Millen, P. F. Kador, and J. H. Kinoshita. 1986. Aldose reductase localization in dog retinal mural cells. Current Eye Res. 5:833-886.

26. Hohman, T. C., C. Nishimura, W. G. Robison, Jr., and J. H. Kinoshita. 1986. Aldose reductase in cultured human and canine retinal capillary pericytes. Invest. Ophthalmol. Visual Sci. 26:328.

27. Engerman, R. L., and T. S. Kern. 1984. Experimental galactosemia produces diabetic-like retinopathy. Diabetes. 33:97-100.

28. Kern, T. S., and R. L. Engerman. 1985. Hexitol production by canine retinal microvessels. Invest. Ophthalmol. Visual Sci. 26:382384.

29. Robison, W. G., Jr., P. F. Kador, Y. Akagi, J. H. Kinoshita, R. Gonzales, and D. Dvornik. 1986. Prevention of basement membrane thickening in retinal capillaries by a novel aldose reductase inhibitor, tolrestat. Diabetes. 35:295-299.

30. Cunha-Vaz, J. G., C. C. Mota, E. C. Leite, J. R. Abreu, and M. A. Ruas. 1986. Effect of Sorbinil on blood-retinal barrier in early diabetic retinopathy. Diabetes. 35:574-578.
31. Gabbay, K. H. 1973. Role of sorbitol pathway in neuropathy. Adv. Metab. Disord. 417-424.

32. Griffey, R., R. P. Eaton, C. Gaspavoric, and W. Sibbit. 1987. Galactose neuropathy. Diabetes. 36:776-778.

33. Tomlinson, D. R., P. R. Holmes, and J. H. Mayer. 1982. Reversal, by treatment with an aldose reductase inhibitor, of impaired axonal transport and motor nerve conduction velocity in experimental diabetes mellitus, Neurosci. Lett. 31:189-193.

34. Yue, D. K., M. A. Hanwell, P. M. Satchell, and J. R. Turtle. 1982. The effect of aldose reductase inhibition on motor nerve conduction velocity in diabetic rats. Diabetes. 31:789-794.

35. Judzewitsch, R., J. B. Jaspan, K. S. Polonsky, C. R. Weinberg, J. B. Halter, E. Halar, M. A. Pfeifer, C. Vukadinovic, L. Bernstein, and M. Schneider, et al. 1983. Aldose reductase inhibition improves motor nerve conduction velocity in diabetic patients. N. Engl. J. Med. 308:119-125.

36. Sima, A. A. F., V. Bril, V. Nathanial, and D. A. Greene. The effect of sorbinil treatment on diabetic neuropathy. Exerpta Med. Congr. Ser. In press.

37. Fagius, J., and S. Jameson. 1981. Effects of aldose reductase inhibitor treatment in diabetic polyneuropathy: a clinical and neurophysiological study. J. Neurol. Neurosurg. Psychiatry. 44:991-1001.

38. Young, R. J., D. J. Ewing, and B. F. Clarke. 1983. A controlled trial of sorbinil, an aldose reductase inhibitor, in chronic painful diabetic neuropathy. Diabetes. 32:938-942.

39. Jaspan, J. B., V. L. Towle, R. Maselli, and K. Herold. 1986. Clinical studies with an aldose reductase inhibitor in the autonomic and somatic neuropathies of diabetes. Metab. Clin. Exp. 35:83-92.

40. Pfeifer, M., H. Snider, H. Peterson, J. Cyrus, and V. Broadstone. Improvement in sympathetic nervous system and cardiovascular performance by sorbinil in diabetics. Exerpta Med. Congr. Ser. In press.

41. Seland, J. H., and L. T. Chylack. 1986. Acute glucose-derived osmotic stress in rabbit lens. Acta Ophthalmol. 64:533-539.

42. Chylack, L. T., W. Tung, and R. Harding. 1986. Sorbitol production in the lens: a means of counteracting glucose-derived osmotic stress. Ophthalmic Res. 18:313-320. 